\title{
THE MOMENTS OF THE RIESZ-NÁGY-TAKÁCS DISTRIBUTION OVER A GENERAL INTERVAL
}

\author{
In SOO BAEK
}

\begin{abstract}
In this paper, the moments of the Riesz-Nágy-Takács(RNT) distribution over a general interval $[a, b] \subset[0,1]$, are found through the moments of the RNT distribution over the unit interval, $[0,1]$. This is done using some special features of the distribution and the fact that $[0,1]$ is a self-similar set in a dynamical system generated by the RNT distribution. The results are important for the study of the orthogonal polynomials with respect to the RNT distribution over a general interval.
\end{abstract}

\section{Introduction}

A distribution $F(x)$ is a bounded, non-decreasing function on the unit interval $[0,1]$ with an infinite set of points of increase $[2,5]$. The $n$-th moment $c_{n}=c_{n}(F)$ of a distribution $F(x)$ is then given by the Riemann-Stieltjes integral [9, p. 122]

$$
c_{n}=\int_{0}^{1} x^{n} d F(x), \quad n=0,1,2, \ldots
$$

As discussed in $[2,5]$, the moments of a distribution are crucial to the investigation of monic orthogonal polynomials over the unit interval $[0,1]$ with respect to the distribution, since the coefficients of such polynomials are determined by these moments. In particular, the asymptotic behavior of the coefficients depends on that of the moments.

Starting in the early 1990s, several papers $[4,5,6,7]$ appeared on the moments of singular distributions, the prototypical case being that of the Cantor distribution. Recently we [2] gave a recurrence formula to find the moments of the RNT distribution [8] which is a strictly increasing singular function on the unit interval, whereas the dyadic case of the RNT distribution was considered in [5].

Received January 15, 2009; Revised April 26, 2009.

2000 Mathematics Subject Classification. Primary 26A30; Secondary 28A80.

Key words and phrases. Riemann-Stieltjes integral, moment, interval of orthogonality, singular distribution function, metric number theory.

This work was supported by the Korea Research Foundation Grant funded by the Korean Government(MOEHRD, Basic Research Promotion Fund)(KRF-2008-521-C00006). 
Another important topic for the orthogonal polynomials with respect to the distribution is the study of orthogonal polynomials over a general interval different from the unit interval. We note that every interval $[\alpha, \beta]$ in the unit interval can be decomposed into a countable union of some essentially disjoint fundamental intervals appearing in the construction of the self-similar set $[0,1]$ [1], [3, p. 35]. So we only need to find the formulas of the Riemann-Stieltjes integrals with respect the RNT distribution over the fundamental intervals. In this paper, we find the general formulas of the Riemann-Stieltjes integrals with respect the RNT distribution over the fundamental intervals using the so-called $(\tau, \tau-1)$-expansion [8] of the unit interval. In particular, we give the general formula for a positive integer $i$

$$
\int_{\gamma}^{\delta} x^{i} d F(x)=C \int_{0}^{1}(A x+B)^{i} d F(x),
$$

where $A, B, C$ are constants depending on the fundamental interval $[\gamma, \delta]$. Thus, the Riemann-Stieltjes integral $\int_{\gamma}^{\delta} x^{i} d F(x)$ with respect to the RNT distribution $F$ over the fundamental interval $[\gamma, \delta]$ is a linear combination of its moments $c_{j}=\int_{0}^{1} x^{j} d F(x)$ with integers $0 \leq j \leq i$. This means that essentially the moments of the RNT distribution over the unit interval give the value of

$$
\int_{\alpha}^{\beta} P(x) d F(x)
$$

of a polynomial $P(x)$ with respect to the RNT distribution $F$ over $[\alpha, \beta]$. Finally we give concrete and non-trivial examples and some useful application for these moments.

\section{Preliminaries}

Let $\mathbb{N}$ be the set of the positive integers. For $a \in(0,1)$ and $p \in(0,1)$, we recall the Riesz-Nágy-Takács(RNT) distribution $[2,8] F=F_{a, p}$ defined on $[0,1]$ by $F(0)=0$ and

$$
F(x)=\sum_{j=1}^{\infty} \frac{(1-p)^{j-1}}{p^{j-1}} p^{m_{j}}
$$

for

$$
x=\sum_{j=1}^{\infty} \frac{(1-a)^{j-1}}{a^{j-1}} a^{m_{j}} \in(0,1]
$$

with integers $1 \leq m_{1}<m_{2}<\cdots<m_{j}<\cdots$. We note that if $a \neq p$, $F_{a, p}$ is a singular function whereas it is the identity function if $a=p$. We [2] already know that the $j$-th moment $c_{j}$ of the RNT distribution $F_{a, p}$ satisfies 
the recurrence relation

$$
\left[1-a^{j} p-(1-p)(1-a)^{j}\right] c_{j}=(1-p) \sum_{l=0}^{j-1}\left(\begin{array}{l}
j \\
l
\end{array}\right) a^{j-l}(1-a)^{l} c_{l}
$$

for each $j \in \mathbb{N}$ where $\left(\begin{array}{l}j \\ l\end{array}\right)=\frac{j !}{l !(j-l) !}$.

We define the $n$-th fundamental interval $I_{i_{1} \cdots i_{n}}=f_{i_{1}} \circ \cdots \circ f_{i_{n}}(I)$ where $f_{0}(x)=a x$ and $f_{1}(x)=a+(1-a) x$ on $I=[0,1], i_{j} \in\{0,1\}$ and $1 \leq j \leq n$, $n \in \mathbb{N}$. Clearly there are $2^{n} n$-th fundamental intervals in $[0,1]$. We note that $[0,1]$ is the self-similar set by the iteration function system $\left\{f_{0}, f_{1}\right\}[3$, p. 29] satisfying the open set condition.

\section{Main results}

We now give the translation and scaling properties of the RNT distribution $F$. The essential properties are given in [2]. The following theorem is a generalization of the Theorem 1 of [2]. By $F$ we denote $F_{a, p}$.

Theorem 1. For $a \in(0,1), p \in(0,1), n \in \mathbb{N}$ and $x \in[0,1]$, we have

$$
F\left(\gamma+a^{n-k}(1-a)^{k} x\right)=F(\gamma)+p^{n-k}(1-p)^{k} F(x),
$$

where $\left[\gamma, \gamma+a^{n-k}(1-a)^{k}\right]$ is the $n$-th fundamental interval where $k=0,1, \ldots, n$.

Proof. It follows immediately from the iterative manipulation of (4) and (5) of Theorem 1 of [2].

We give the general formula of the Riemann-Stieltjes integral

$$
\int_{\alpha}^{\beta} \phi(x) d F(x)
$$

of a continuous function $\phi$ with respect to $F$ over $[\alpha, \beta]$ from the translation and scaling properties (7) of the RNT distribution whereas we already gave the essential formulas (6) and (7) in [2]. The following Theorem is a generalization of Theorem 2 of [2].

Theorem 2. Let $a_{n, k}=a^{n-k}(1-a)^{k}$ where $k=0,1, \ldots, n$ for $n \in \mathbb{N}$. For a continuous function $\phi$ and each $n \in \mathbb{N}$, we have the following $2^{n}$ equations such that

$$
\int_{\gamma}^{\gamma+a_{n, k}} \phi(x) d F(x)=p^{n-k}(1-p)^{k} \int_{0}^{1} \phi\left(\gamma+a_{n, k} x\right) d F(x),
$$

where $\left[\gamma, \gamma+a_{n, k}\right]$ is an $n$-th fundamental interval.

Proof. Let $m \in \mathbb{N}$ and $0 \leq i \leq m$, let $x_{i}=\gamma+i a_{n, k} / m$. So we have the partition

$$
\gamma=x_{0}<x_{1}<x_{2}<\cdots<x_{m}=\gamma+a_{n, k}
$$


of $\left[\gamma, \gamma+a_{n, k}\right]$. Writing $y_{i}=\left(x_{i}-\gamma\right) / a_{n, k}$, we convert this partition into one of $[0,1]$ :

$$
0=y_{0}<y_{1}<y_{2}<\cdots<y_{m}=1 \text {. }
$$

Consequently, combining (7) with the definition of the Riemann-Stieltjes integral, yields

$$
\begin{aligned}
& \int_{\gamma}^{\gamma+a_{n, k}} \phi(x) d F(x)=\lim _{m \rightarrow \infty} \sum_{i=1}^{m} \phi\left(x_{i}\right)\left[F\left(x_{i}\right)-F\left(x_{i-1}\right)\right] \\
= & \lim _{m \rightarrow \infty} \sum_{i=1}^{m} \phi\left(\gamma+a_{n, k} y_{i}\right)\left[F\left(\gamma+a_{n, k} y_{i}\right)-F\left(\gamma+a_{n, k} y_{i-1}\right)\right] \\
= & \lim _{m \rightarrow \infty} \sum_{i=1}^{m} \phi\left(\gamma+a_{n, k} y_{i}\right) p^{n-k}(1-p)^{k}\left[F\left(y_{i}\right)-F\left(y_{i-1}\right)\right] \\
= & p^{n-k}(1-p)^{k} \int_{0}^{1} \phi\left(\gamma+a_{n, k} y\right) d F(y) .
\end{aligned}
$$

This establishes (8).

Remark 1 . In the above theorem, the $n$-th fundamental interval $\left[\gamma, \gamma+a_{n, k}\right]$ is of the form $I_{i_{1} \cdots i_{n}}=f_{i_{1}} \circ \cdots \circ f_{i_{n}}(I)$ as we recalled in the preliminaries, that is, $\gamma=f_{i_{1}} \circ \cdots \circ f_{i_{n}}(0)$ and $\gamma+a_{n, k}=f_{i_{1}} \circ \cdots \circ f_{i_{n}}(1)$ for some $i_{j} \in\{0,1\}$ and $1 \leq j \leq n$. From now on, we denote $a^{n-k}(1-a)^{k}$ by $a_{n, k}$.

Theorem 3. Let $i$ be a non-negative integer. For the $n$-th fundamental interval $[\gamma, \delta]=\left[\gamma, \gamma+a_{n, k}\right]$ for some integer $0 \leq k \leq n$, we have

$$
\int_{\gamma}^{\delta} x^{i} d F(x)=p^{n-k}(1-p)^{k} \sum_{j=0}^{i}\left(\begin{array}{l}
i \\
j
\end{array}\right) a_{n, k}^{j} \gamma^{i-j} c_{j},
$$

where $c_{j}$ is the $j$-th moment of the RNT distribution $F$.

Proof. From (8),

$$
\begin{aligned}
\int_{\gamma}^{\delta} x^{i} d F(x) & =\int_{\gamma}^{\gamma+a_{n, k}} x^{i} d F(x) \\
& =p^{n-k}(1-p)^{k} \int_{0}^{1}\left(a_{n, k} x+\gamma\right)^{i} d F(x) \\
& =p^{n-k}(1-p)^{k} \sum_{j=0}^{i}\left(\begin{array}{l}
i \\
j
\end{array}\right) a_{n, k}^{j} \gamma^{i-j} \int_{0}^{1} x^{j} d F(x) \\
& =p^{n-k}(1-p)^{k} \sum_{j=0}^{i}\left(\begin{array}{l}
i \\
j
\end{array}\right) a_{n, k}^{j} \gamma^{i-j} c_{j} .
\end{aligned}
$$

This establishes (9). 
Remark 2. In (2) we stated that

$$
\int_{\gamma}^{\delta} x^{i} d F(x)=C \int_{0}^{1}(A x+B)^{i} d F(x),
$$

where $A, B, C$ are constants depending on the fundamental interval $[\gamma, \delta]$. In the proof of the above theorem, we see that $C=p^{n-k}(1-p)^{k}, A=a_{n, k}=$ $a^{n-k}(1-a)^{k}$ and $B=\gamma$, which depend on the fundamental interval $[\gamma, \delta]=$ $\left[\gamma, \gamma+a_{n, k}\right]$. It is sometimes easier to use (2) than (9) for the calculation of the moments over the fundamental interval.

Example 1. For $a=1 / 4$ and $p=1 / 2$, we easily have $c_{0}=1, c_{1}=\frac{1}{4}, c_{2}=\frac{5}{44}$ and $c_{3}=\frac{278}{4400}$ from $(6)$.

From (2) and the above remark, we have

$$
\int_{\gamma}^{\gamma+(1 / 4)^{n-k}(3 / 4)^{k}} x^{i} d F(x)=\frac{1}{2^{n}} \int_{0}^{1}\left((1 / 4)^{n-k}(3 / 4)^{k} x+\gamma\right)^{i} d F(x),
$$

where $\left[\gamma, \gamma+(1 / 4)^{n-k}(3 / 4)^{k}\right]$ is one of the $n$-th fundamental intervals.

In particular, for $i=3$ and $n=2$,

$$
\begin{aligned}
\int_{0}^{(1 / 4)^{2}} x^{3} d F(x) & =\frac{1}{2^{2}} \int_{0}^{1}\left((1 / 4)^{2} x+0\right)^{3} d F(x)=\frac{c_{3}}{2^{2} \cdot 4^{6}} \\
\int_{(1 / 4)^{2}}^{(1 / 4)^{2}+(1 / 4)(3 / 4)} x^{3} d F(x) & =\frac{1}{2^{2}} \int_{0}^{1}\left((1 / 4)(3 / 4) x+(1 / 4)^{2}\right)^{3} d F(x) \\
& =\frac{3^{3} c_{3}}{2^{2} \cdot 4^{6}}+\frac{3 \cdot 3^{2} c_{2}}{2^{2} \cdot 4^{6}}+\frac{3 \cdot 3^{1} c_{1}}{2^{2} \cdot 4^{6}}+\frac{c_{0}}{2^{2} \cdot 4^{6}} \\
x_{(1 / 4)}^{(1 / 4)+(1 / 4)(3 / 4)} x^{3} d F(x)= & \frac{1}{2^{2}} \int_{0}^{1}((1 / 4)(3 / 4) x+(1 / 4))^{3} d F(x) \\
= & \frac{3^{3} c_{3}}{2^{2} \cdot 4^{6}}+\frac{3 \cdot 3^{2} \cdot 4 c_{2}}{2^{2} \cdot 4^{6}}+\frac{3 \cdot 3^{1} \cdot 4^{2} c_{1}}{2^{2} \cdot 4^{6}}+\frac{4^{3} c_{0}}{2^{2} \cdot 4^{6}}, \\
\int_{1-(3 / 4)^{2}}^{1} d F(x)= & \frac{1}{2^{2}} \int_{0}^{1}\left((3 / 4)^{2} x+\left(1-(3 / 4)^{2}\right)\right)^{3} d F(x) \\
= & \frac{3^{6} c_{3}}{2^{2} \cdot 4^{6}}+\frac{3 \cdot 3^{4}\left(4^{2}-3^{2}\right) c_{2}}{2^{2} \cdot 4^{6}}+\frac{3 \cdot 3^{2}\left(4^{2}-3^{2}\right)^{2} c_{1}}{2^{2} \cdot 4^{6}} \\
& +\frac{\left(4^{2}-3^{2}\right)^{3} c_{0}}{2^{2} \cdot 4^{6}} .
\end{aligned}
$$

Example 2. In the above example, that is for $a=1 / 4$ and $p=1 / 2$, we already had $c_{0}=1, c_{1}=\frac{1}{4}, c_{2}=\frac{5}{44}$. From (9) with $1 / 13=\sum_{k=2}^{\infty}(1 / 4)^{k}(3 / 4)^{k-2}$, we have

$$
\int_{0}^{1 / 13} x^{2} d F(x)=\frac{1}{8^{2}}\left(\begin{array}{l}
2 \\
2
\end{array}\right) c_{2}+\sum_{n=2}^{\infty} \frac{1}{8^{2 n}} \sum_{j=0}^{2}\left(\begin{array}{l}
2 \\
j
\end{array}\right) 3^{(n-1) j} \sum_{k=2}^{n}\left((1 / 4)^{k}(3 / 4)^{k-2}\right)^{2-j} c_{j} .
$$


It is a non-trivial example of the moment of the RNT distribution $F=$ $F_{1 / 4,1 / 2}$ over a general interval $[0,1 / 13]$ which is not a fundamental interval.

Remark 3. The well-known Stone-Weierstrass theorem [9, p. 159] implies that if $\phi$ is a continuous function on an interval $[\alpha, \beta]$, then there is a sequence of polynomials $P_{k}$ such that

$$
\lim _{k \rightarrow \infty} P_{k}(x)=\phi(x)
$$

uniformly on $[\alpha, \beta]$. Clearly the Riemann-Stieltjes integral

$$
\int_{\alpha}^{\beta} \phi(x) d F(x)
$$

of a continuous function $\phi$ with respect to the RNT distribution $F$ over $[\alpha, \beta]$ can be represented by

$$
\sum_{n=1}^{\infty} \int_{\gamma_{n}}^{\delta_{n}} \phi(x) d F(x),
$$

where $\left[\gamma_{n}, \delta_{n}\right]$ are some fundamental intervals of the self-similar set $[0,1]$. Further the Riemann-Stieltjes integral of $\phi$ over the fundamental interval $[\gamma, \delta]$

$$
\int_{\gamma}^{\delta} \phi(x) d F(x)=\lim _{k \rightarrow \infty} \int_{\gamma}^{\delta} P_{k}(x) d F(x)
$$

for a sequence of polynomials $P_{k}$ from the Stone-Weierstrass theorem. This implies that essentially the moments of the RNT distribution over the unit interval also give the value of

$$
\int_{\alpha}^{\beta} \phi(x) d F(x)
$$

of a continuous function $\phi(x)$ with respect to the RNT distribution $F$ over $[\alpha, \beta]$.

Acknowledgement. The author thanks a referee for revising this paper.

\section{References}

[1] I. S. Baek, Dimensions of distribution sets in the unit interval, Commun. Korean Math. Soc. 22 (2007), no. 4, 547-552.

[2] _ A note on the moments of the Riesz-Nágy-Takács distribution, J. Math. Anal. Appl. 348 (2008), no. 1, 165-168.

[3] K. J. Falconer, Techniques in Fractal Geometry, John Wiley \& Sons, Ltd., Chichester, 1997.

[4] H. Fischer, On the paper: "Asymptotics for the moments of singular distributions" [J. Approx. Theory 74 (1993), no. 3, 301-334] by W. Goh and J. Wimp, J. Approx. Theory 82 (1995), no. 3, 362-374.

[5] W. Goh and J. Wimp, Asymptotics for the moments of singular distributions, J. Approx. Theory 74 (1993), no. 3, 301-334.

[6] P. J. Grabner and H. Prodinger, Asymptotic analysis of the moments of the Cantor distribution, Statist. Probab. Lett. 26 (1996), no. 3, 243-248. 
[7] F. R. Lad and W. F. C. Taylor, The moments of the Cantor distribution, Statist. Probab. Lett. 13 (1992), no. 4, 307-310.

[8] J. Paradís, P. Viader, and L. Bibiloni, Riesz-Nágy singular functions revisited, J. Math. Anal. Appl. 329 (2007), no. 1, 592-602.

[9] W. Rudin, Principles of Mathematical Analysis, Third edition. International Series in Pure and Applied Mathematics. McGraw-Hill Book Co., New York-Auckland-Dusseldorf, 1976.

Department of Mathematics

Pusan University of Foreign Studies

PUsAn 608-738, KoreA

E-mail address: isbaek@pufs.ac.kr 